\title{
The Guiding Role of Animation Plots in Cognitive Awareness during Children's Growth*
}

\author{
Qingke Liu \\ Nanjing University of Posts and Telecommunications \\ Nanjing, China
}

\begin{abstract}
Animation plays an indelible role in children's growth. This article aims to explore the guiding role of Chinese cartoons in children's growth from the perspective of children and discover the problems of our country's cartoons through the comparison of the domestic animation and The Pig Peggy, and then provides more practical recommendations to our country's animation industry.
\end{abstract}

Keywords-animation; animation education; children's cognition; guiding role

\section{INTRODUCTION}

When it comes to cartoons, everyone has a unique memory. They have brought so many people incessant wonderful time and memories. However, can animation play a better role in guiding children's cognitive behavior? "If the youth are strong, the country will be strong." The creation of animation has an inescapable responsibility for the cultural education for children and adolescents. Therefore, we should pay attention to the education-oriented role of the animation, and enable children to truly integrate themselves into the excellent cultures from childhood. Accordingly, we enlarge the effect of the animation to different age levels so as to let cartoons not only simply teach through lively activities but also become a carrier of traditional Chinese culture.

In this study, a total of 503 online questionnaires are distributed to and collected from parents of school-aged children $(0-6$ years old $)$

\section{THE ROLE OF ANIMATION IN CHILDREN'S GROWTH}

\section{A. The Companionship in Children's Lives}

In childhood animations cannot be neglected apart from the companionship of family members and toys. In the process of watching the cartoons children go deep into the plots of the stories to their heart's content. Therefore, many parents regard animations as a "safe haven" for them to supervise their children.

\section{B. The Expansion of Children's Horizons}

Through the dialogues between the characters and the emergence of the plots in the animations, children can be deeply involved in the event to learn to analyze and handle some problems. Take the Big Ear Tutu for example; new

*Project funded: No.JG00816JX83; No. JG30116006

\author{
Keyu Guo \\ Nanjing University of Posts and Telecommunications \\ Nanjing, China
}

problems appear in every part. Then at the end some solutions will be presented. The classic animation column Big Windmill has attracted the attention of countless children when it is broadcast on CCTV in the prime time. The Jigsaw Puzzle and The Smart House especially enable the common sense of life and scientific knowledge easy to understand. By means of the combination of interestingness and the animated characters children acquire new knowledge while watching the cartoons.

\section{The Adjustment to Children's Emotion and the Exploitation of Children's Potential}

The cartoons are remarkable for both voice and expression. The plots are filled with joy and affections. When children watch the cartoons, their pleasure, anger, sorrow, and joy are stimulated. They can experience the real happiness. Therefore, the animations will help to tap into children's areas of interest and discover some interesting details and phenomena in their lives. In addition, they contribute to arousing children's love of things around them and kindling their interest in participating in some activities and cultivating their exploring spirit as well as enhancing their imagination.

\section{The Guidance on Children's Values}

The cartoons help children to cultivate correct values. Since the animations usually present kind and beautiful images, such as the Pleasant Goat in the Pleasant Goat and Big Wolf, who is full of wisdom and justice? Children approve of them. Consequently, the shaping of the characters is conducive to forming correct values for children when they start to understand the world. Children will begin to advocate justice and be braver. Therefore, animation plays a huge role in guiding children's values in their growth.

\section{E. The Improvement in Children's Language Competence}

Children are at a special age stage where they mainly imitate and learn from others, especially as regards language. According to the interviews with the parents of some children, they make it known that children's imitativeness of the characters as well as their language in the animations is very intense. They can learn a great number of fresh nouns and words quickly.

On the other hand, "animation has a negative effect on children in certain aspects as well." To start with, the act of 
violence in the cartoons causes an incorrect guidance on children. For example, in the Boonie Bears Guangtouqiang usually solves problems through violence. Since children have a strong ability to imitate others at their young age, it is inevitable that they have strong imitative tendencies toward the behavior of the characters. In the meantime, they lack the ability to tell right from wrong. It is easy for them to garble a statement. In this case, the guardians should undertake more responsibility. In addition, animations tend to increase children's unrealistic fantasies about reality. Some time ago, the article on how much money Peggy's life needed was heated. There was a big difference for Peggy's vehicles and toys in comparison with those in reality. Finally, the animation induces children to form an incorrect career outlook. For example, many children find that doctors are always serious and even horrible when they enjoy the cartoons. As a result, they will misunderstand the profession of doctors.

\section{THE ROLE OF ANIMATION IN GUIDING CHILDREN}

\section{A. Cultural Orientation}

A successful animation is not only remarkable for its plot but also reflective of the cultural background. The Pig Peggy reflects the British education and culture, which advocate equality for all. Even if the siblings work together, parents rarely convey any stereotyped value guidance that "elder sisters should give the priority to their brothers". This kind of education advocates they should communicate with each other and settle the problems for the sake of the other side. In the family what should be presented is a free and equal environment. On the contrary, in the Boonie Bears, we find that Briar, the elder bear, always takes advantage of his age to exert mandatory requirements on Bramble while the latter bear also comes up with an idea of relying on his brother when he is faced with difficulties. China is accustomed to the traditional cultural concept of hierarchy and inferiority. Therefore, we need the guidance of the free and equal values.

The animation film Mulan by Disney depicts an image which lays stress on self-esteem. It is greatly distinguishable from the image of Mulan in our hearts. This precisely illustrates that the animations differ under the influence of the political and cultural orientation in different countries. As mentioned in the previous research papers: "the mighty culture of the American animation conveyance not only has a strong spread capacity but also strongly expands its cultural identity and advocates its domineering status. Ours belongs to weak culture. But the strength of the culture does not represent its pros and cons."

\section{B. Cognitive Orientation}

Children's values are easily influenced by the surrounding things. In addition to their parents' teachings and deeds, the animations become the second door to their understanding of the world because they tell the truth that many parents may neglect. Therefore, parents are more willing to let their children enrich their knowledge and broaden their horizons by watching cartoons. However, different contents have different guiding functions. Not all animations are suitable for children at a certain age. The Boonie Bears mainly talks about a series of stories that aim to protect the forest ecology. However, it is not possible for school children to fight with those who overexploit the forest in the park. Meanwhile, a series of vulgar words, such as "provoke me, I will beat you without doubt," "shut up," "dream," are easy for children to learn. Compared with The Boonie Bears, The Pig Peggy mainly promotes affections and friendships. This cartoon teaches children how to get along with their family and deal with difficult issues as well as how to get along with others in a happy manner. These are what children really need to learn. Through the animation they can feel the same situation. They will be able to get along better with others. Besides, in other Chinese cartoons, there are also problems of delivering incorrect cognitive orientation. For example, the beating and killing in the Pleasant Goat and Big Wolf are detrimental to children while the British cartoon Thomas and His Friends conveys a core value of "being a valuable person." Each episode embodies the joy of helping others and solving difficulties.

\section{Behavior Orientation}

The director of The Boonie Bears said: "this cartoon aims to teach through lively activities the positive values that friendship is precious but the domestic affection is higher". We can't deny his original intention. But the contents of the animation are really not satisfactory. The Pig Peggy also conveys the same kind of affections and friendships. But children can learn through the animation how Peggy gets along with her good friends and faces specific difficulties. The piglets in The Pig Peggy always like to compete with the mud pit. In the process of communicating with parents we find that at first this "jumping pit" phenomenon causes their dissatisfaction. They report that their children are keen to jump into the mud pit and splash water. But before long the British producers made the fastest improvement. Peggy's mother said that she must wear raincoats and rain boots before she can play. This modification was then praised by all parents. It can be seen that every detail in the animation may be a "model role" for imitation and learning. For the children at their early age we must not only have an educational-oriented original intention but also have some seemingly simple and tedious details to help to convey our main ideas.

In China there are some prominent educative animation columns, such as Little Tree of Wisdom, Big Windmill and so on. But generally speaking, the number is comparatively small and the contents are not abundant. As a result, this kind of column is not very popular. But in the West, in terms of the United States, "multiple intelligent teaching animations such as Blue's Clues, Mickey Mouse club, Pooh Big Detective and Little Einstein emerge endlessly." The interactive animation design not only enhances the connection between parents and children but also promotes children's understanding of relevant knowledge. Many parents account this kind of animation a compulsory course in their children's growth. Additionally, the traditional Chinese "cramming" education model is also reflected in the animations. For example, the ending of the Big-ear Tutu 
educates the children through teaching or draws children's attention through Tutu's parents' education on him. But children's real acceptance rate is not high. In the United States the philosophical theory contained in some simple animations is hidden in the performance of the characters. Children can explore the truth through watching the lively programs.

Through the analysis of the data we find that parents think about half effect of the edutainment animations has been fulfilled. Parents are willing to act as a bridge between the animations and children. Yet they explain little. Through the analysis of regression, we find that there is a correlation between the extent to exert teaching through lively activities and parent's explanation to children $(\mathrm{Sig}<0.05)$. Therefore, when the educational function of teaching through lively activities cannot be fully realized, this responsibility must fall on the parents' shoulders. But through the interviews with the parents, we find that many parents still have a lot of realistic pressure that makes it difficult to concentrate on accompanying their children and making presentations to them.

\section{REFLECTIONS AND SUGGESTIONS ON THE DEVELOPMENT OF CHINESE ANIMATION IN THE FUTURE}

\section{A. Get Rid of the Preaching Mode and Respect Children's Cognitive Characteristics}

The animation plays a significant role in education. However, if it merely permeates the culture of the nation through a preaching mode and neglects the self-development that children really need, it will eventually lose the trust of children and parents. China's children's animation should continue to take the path of innovation in national culture and inherit the profoundness of traditional culture as well as integrate national culture with the animation organically instead of preaching simply. Then our animation is able to achieve a win-win result of both entertainment value and education value.

\section{B. Improve the Quality of the Animation}

A classic animation not only accompanies our childhood but also represents our childhood. This does show the importance of an excellent animation work. Children's animation should establish a child-oriented creative concept, reducing vulgar contents. Thus, the creation of children's animation should first consider their cognitive ability of comprehension. Then it should be on the basis that the fundamental role of animation is to bring children joy and attract their attention. Therefore, whether in the setting of the plots or in the arrangement of the characters the contents must be more abundant and the plots must be more interesting. Besides, the relevant government departments should strengthen the censorship of animations, making the works update slower so as to guarantee their quality.

\section{Learn from Others' Advantages for Self-improvement}

Every time after watching some western excellent animations a heated online discussion about the gap between
Chinese and Western animation and culture will arise. The traditional Chinese culture is extensive and profound. We should tap into our excellent traditional culture, obtaining its essence and eliminating its dregs. Then our children's animation is able to reflect our core cultural value orientation. However, the current domestic animation rarely shows our unique traditional cultural features. Instead, it repeatedly "chews" the culture that should be eliminated. Now is the era of economic globalization. In addition to the flow of related industries where the economy is the main body, the animation is constantly "bringing in and going out". What we can do is not simply to promote or resist a certain type of animation. In contrast, we should reflect on our own animation and enhance its competitiveness.

Therefore, apart from carrying forward the traditional culture, our domestic animation still needs to absorb the essence of the times. It should possess the awareness of the times and the vision of the world with the infusion of our own humanistic spirit and finally achieve the perfect combination of the tradition and the times as well as the Chinese parts and the Western parts, especially in some children's cartoons. We should learn the forms and methods that are taken advantage of to exert the educational effect of the children's animation in the west and innovate continuously so as to fulfill the role of our domestic animation in children's education to the full.

\section{Define the Entertaining Feature of Children's Animation}

We have found that many animations are not childoriented creations. The connotation is based on the perspective of adults. We cannot fully conclude that children's precocity is entirely due to the animation, as a medium of knowledge and culture transmission, words and deeds in the cartoons will inevitably affect children directly. According to a survey, $87.9 \%$ of parents think it is necessary that violence in the cartoons should be removed at least for young children. $87.1 \%$ of parents think that the connection between the characters in the cartoons and the real life should be strengthened so as to make initial preparations for children to understand the world and enter the society in the future. The establishment of certain professional roles and the division of role between men and women should be more rigorous. As a carrier of children's life and happiness, animation cannot be neglected. We should fully be aware that animation should see children as the main body, considering from the perspective of children to achieve the purpose of "being happy" by making children pleased; animation should spread the corresponding scientific and cultural knowledge and explain the truths in a straightaway and understandable way so as to attain the goal of "education" and thus really play a vital role in the education of children. At the same time, the contents of animation should be appropriately graded so that parents can better select the cartoons suitable to some age-appropriate children and ultimately promote the educational significance of the animation. 


\section{CONCLUSION}

Children are the successors of the future and the pillars of our motherland. To foster children, we cannot totally transfer the responsibility to teachers and parents. Animations serve as a "good friend" of children in the process of their growth. Therefore, we should also pay attention to its educational and guiding effect on children. Our domestic animation has entered a period of rapid development. I firmly believe that our animation industry will get rid of the stale and bring forth the fresh and make advances and improvements continuously. In the future there must be a glittering field that belongs to animation and the animation industry will become a representative of our country's culture.

\section{REFERENCES}

[1] Li Yanping, Ma Guansheng watching TV on cognition and behavior of children and juveniles [J]. Foreign Medicine (Hygienics Volume), 2002 (3): 157-161.

[2] Li Wei, A Comparative Study on the Characteristics of the Types of Chinese Kindergarten Cartoons and Their Educational Values[D]. Master thesis of Shaanxi Normal University, 2015

[3] Shi Liumei. Differences in the influence of Chinese and English cartoons on adolescents' values_- Taking "Bear Infestation" and "Thomas and His Friends" as Examples [J]. Audio-visual World, 2015, (02): 92-94.

[4] Du Simao. May positive energy domestic animation accompany the growth of Chinese children[N]. China Film Weekly:2014

[5] Li Wei, A Comparative Study on the Characteristics of the Types of Chinese Kindergarten Cartoons and Their Educational Values[D]. Master thesis of Shaanxi Normal University, 2015 